Hernia

https://doi.org/10.1007/s10029-018-1833-x

\title{
Long-term outcomes after prophylactic use of onlay mesh in midline laparotomy
}

\author{
C. San Miguel1 · D. Melero1 - E. Jiménez1 - P. López1 - Á. Robin1 · L. A. Blázquez1 · J. \\ López-Monclús2 · E. González1 • \\ C. Jiménez1 • M. Á. García-Ureña1
}

\section{INTRODUCTION}

Incisional hernias $(\mathrm{IH})$ are a frequent complication of abdominal wall incisions. They occur in 5$20 \%$ of the general patient population [1-3]. In high-risk patients, the incidence of IH can increase to more than $60 \%[4,5]$. In the emergency setting, the reported incidence of $\mathrm{IH}$ is greater than $50 \%[6,7]$. IH can cause morbidity and have a negative effect on patients' quality of life (QoL). Despite advances in IH repair, recurrence rates remain high (12-54\%) [8], and those who experience recurrence are susceptible to a vicious cycle of complications, as each subsequent repair presents a greater technical challenge with an increased risk of further recurrence and morbidity [9]. In terms of healthcare economics, in 2011, a study from France has shown that if the rate of $\mathrm{IH}$ after abdominal surgery could be reduced to $5 \%$, a total of 4 million Euros would have been saved [10]. Therefore, prevention of IH is important for both patients and health care providers [3].

Risk factors for IH development are both patient related and secondary to technical considerations for abdominal wall closure [11]. Some patient related risk factors can be modified to reduce the rate of $\mathrm{IH}$, including smoking cessation, optimizing diabetic control, weight loss 
and implementing bundles to reduce SSI. The technical considerations that can be employed to reduce the incidence of $\mathrm{IH}$ include the choice of suture material, the use of either small bites to close the linea alba and prophylactic mesh placement (PMP) [12].

Previous systematic reviews and meta-analyses have shown that PMP is safe and effective at preventing $\mathrm{IH}[3,13]$. However in the specific setting of $\mathrm{PMP}$, there is minimal data on which is the optimal mesh composition, the best anatomical position to place the mesh within the abdominal wall or the best method of mesh fixation [9]. There is also fear regarding the potential long-term sequelae of PMP, such as chronic seroma, chronic pain, infection or mesh explantation. Additionally, there are no data on how to manage those patients that develop an IH after PMP.

Concerned about this problem, and following our satisfactory short to mid-term results in PMP after midline laparotomies (ML) in colorectal surgery [14], we wanted to investigate what happened to patients undergoing PMP after several years. So, the aim of this study was to evaluate the long-term outcomes after prophylactic use of onlay mesh in ML.

\section{METHODS}

This observational study was conducted in the Department of General and Digestive Surgery of Henares University Hospital in Madrid, Spain, between 2008-2014. Our center is a 250-bed facility that belongs to the Spanish National Health Service and attends over 160,000 population in the periphery of Madrid, with $20 \%$ of immigrants from Eastern Europe. Surgical team comprises 12 surgeons with specialization in general and digestive surgery who were responsible for both elective and emergency operations. 
The primary outcome of the study was to assess the long-term incidence of IH after PMP. Secondary outcome measures were the need for reoperation, chronic infection, chronic seromas, mesh explantation and chronic pain.

From a prospective maintained database, we have identified those patients over 18 years old, operated by ML with PMP, in either an emergency or elective setting with a minimum follow-up of 2 years. The STROBE Statement recommendations were followed [15]. The decision to perform PMP was made by the surgeon responsible for the patient. All patients were informed $\&$ consented prior to surgery.

As previously described [14], abdominal wall closure was performed by a standardised protocol of PMP. Briefly, the linea alba was closed with running sutures of long-term resorbable monofilament USP number 0 or 1 , spaced $1 \mathrm{~cm}$ apart and $1 \mathrm{~cm}$ from the cut edge. A large-pore, medium-density polypropylene mesh (Optilene Mesh Elastic; B. Braun, Melsungen, Germany) was placed in the fascial onlay position. The mesh was $5 \mathrm{~cm}$ wide and the length of the mesh was adapted to the incision with an overlap of $2 \mathrm{~cm}$ at both ends. The meshes were fixed to the anterior rectus sheath with interrupted resorbable sutures $3-4 \mathrm{~cm}$ apart. When an ostomy was placed, the mesh did not reach or cover the stoma site. The subcutaneous tissue was closed by interrupted polyglactin $2 / 0$ stitches fixed to the mesh and a suction drain was left over the mesh. Staples were used for skin closure.

Registered preoperative clinical data included age, sex, body mass index (BMI), American Society of Anesthesiologists (ASA) grade, comorbidities [hypertension, diabetes, chronic obstructive pulmonary disease (COPD), cardiopathy, collagen disease, etc], previous history of cancer and smoking. Intraoperative variables included operative status (elective or emergency), diagnosis, operative procedure, stoma formation and operation time. Patients who developed full thickness abdominal wall dehiscence (evisceration) or required further surgery within the first 30 days post-operatively undergoing removal of the mesh without any new mesh implantation 
were excluded from data analysis. All post-operative surgical site occurrences were included in the analysis. Centers for Disease Control and Prevention definitions of SSI were used [16]. Seroma was defined as a mass or swelling in the wound caused by the localized accumulation of clear serum liquid without SSI signs. Chronic pain was defined as any pain lasting more than 12 weeks [17]. VAS score $>2$ and the need for analgesia were considered as pain. Mesh explantation was defined as a chronic wound infection that required mesh removal. Complications were diagnosed and registered by the surgeons of the department.

Patient follow-up was carried out in the outpatient clinic by clinical examination. Incisional herniation was diagnosed according to EHS definition [18]. Computed tomographic (CT) scan was performed as part of routine surveillance for oncology patients, when there was any clinical uncertainty regarding the presence of an $\mathrm{IH}$, and for investigation of other complaints not related to the previous surgery.

The description of variables and the statistical analysis were performed using the Statistical Package for the Social Sciences (SPSS) program (version 19.0 for Windows). Quantitative variables were expressed as mean and standard deviation, and categorical variables as absolute numbers and percentages. The statistical analysis of the quantitative variables for independent groups was performed with the nonparametric Mann-Whitney $\mathrm{U}$ test. In the statistical analysis of categorical variables, the Pearson $\chi 2$ (Fisher's) test was used. The appearance of IH during follow-up was analyzed with the Kaplan-Meier estimation method and comparative analysis of time-to-event data was performed using the log rank test. Statistical significance was accepted as $\mathrm{p}<0.05$.

\section{RESULTS}


Between 2008-2014, a cohort of 172 patients underwent PMP following ML (Figure 1). These include 96 men (56\%) and 76 women (44\%), with a mean age of 68 years (62-77) and mean BMI of $28.6 \mathrm{~kg} / \mathrm{m}^{2}(25.2-30.8)$.

Comorbidities (summarized in Table 1) were: smoking, 71 (41.3\%) patients; heart disease, 53 (30.8\%) patients; hypertension 94 (54.7\%) patients; COPD, 24 (14\%) patients; diabetes, 38 (22.1\%) patients; obesity, $44(25.6 \%)$ patients; and previous history of cancer, 34 (19.8\%) patients. The most common indication for surgery was uncomplicated colorectal cancer $(70 \%$ of cases). Other diagnoses included ischemia, acute diverticulitis, and bowel obstruction.

The procedures performed included left hemicolectomy/sigmoidectomy, 45(26.2\%) patients; right hemicolectomy, 39 (22.7\%) patients; anterior resection of rectum, 30 (17.4\%) patients; total/subtotal colectomy, 16 (9.3\%) patients; small bowel resection, 9 (5.2\%) patients; abdominoperineal resection, $8(4.7 \%)$ patients; exploratory laparotomy, 12 (7\%) patients; and adhesiolysis, 11 (6.4\%) patients. Emergency surgery was performed in $36(25 \%)$ patients, after having excluded lost of follow-up patients.

There were 145 (85\%) patients with clean-contaminated wounds; 11 (6.4\%) patients with clean wounds; 2 (1.2\%) patients with contaminated wounds and 14 (8.1\%) patients with dirty wounds. Twenty-nine (17\%) patients were given a colostomy, of which 7 (24\%) were temporary; and 19 (11\%) patients had an ileostomy, of which 13 (68.4\%) were temporary. The mean postoperative hospital stay was 11 (range, 7-18) days.

Related with surgical site occurrences, we registered: 17 (9.9\%) patients with a superficial SSI (Clavien-Dindo grade I); 23 (13.4\%) patients with wound seromas (Clavien-Dindo grade I); 9 (5.2\%) patients with organ/space infection (2 Clavien-Dindo grade IIla, 2 grade IIIb, 3 grade IVa and 2 grade IVb); and 2 (1.1\%) patients with deep SSI (Clavien-Dindo type IIIb). In these last two cases the mesh needed to be removed within the first six months after surgery due to chronic 
infection (Figure 2). The bacterial wound culture isolated Pseudomonas aeruginosa and Escherichia Coli, respectively.

Patients were followed up for up to 8 years in the outpatient clinic (Figure 3), with a mean followup of $5 \pm 1.6$ years. Those patients that were not currently followed-up by the surgeon that performed the original surgery were invited to attend the outpatient clinic for an up-to-date clinical examination by the main author (CSM). Most of them have been reviewed during the last two years before the analysis (Figure 3). The estimated freedom from IH is shown in Figure 4. During follow-up, a global mortality of $29(20 \%)$ patients was registered. None of these were thought to be directly related to PMP. A total of $28(16.2 \%)$ patients were lost to follow up, due to migration of foreign patients or other unknown reasons. Patients undergoing reoperation within 30 postoperative days with removal of the mesh without any new mesh implantation were excluded from data analysis. This included 3 (1.74\%) patients with full thickness abdominal wall dehiscence; and 5 (2.9\%) patients requiring early reoperations ( 2 anastomotic leaks after colorectal surgery, 1 intra-abdominal abscess, 1 early haemoperitoneum and 1 case of peritoneal carcinomatosis). Data from 144 patients were used for final analysis.

During follow-up, 8 patients (5.55\%) underwent further surgery. These included a Hartmann's procedure reversal ( 3 patients), metachronous colon cancer ( 2 patients), adhesions causing intestinal obstruction (1 patient), gastric cancer procedure (1 patient) and urologic surgery (1 patient). No hernia was found during these relaparotomies. A dense fibrosis was found to be covering the linea alba and no adhesions were found directly related to the fibrosis or mesh itself (Figure 5 and Video).

After clinical and radiological examination, 13 patients were diagnosed with $\mathrm{IH}$. One patient was diagnosed with an $\mathrm{IH}$ on $\mathrm{CT}$ after a negative clinical exam. The overall incidence of $\mathrm{IH}$ was $9.02 \%$ (13/144) (Table 2). These included 7 emergency and 6 elective cases. The incidence of IH in those patients requiring emergency surgery was $19.4 \%$ and $5.5 \%$ for elective surgery. In those patients 
that developed an $\mathrm{IH}, 8$ were in patients with colorectal cancer. The remaining cases include 3 patients with non-cancer related bowel obstruction, 1 exploratory laparotomy due to sepsis secondary to duodenal ulcer and 1 acute diverticulitis, requiring a Hartmann's procedure. The incidence of IH in non-cancer patients was $16.1 \%$ (5/31 patients) and 7\% (8/113 patients) in the oncological group.

In those patients that developed an $\mathrm{IH}, 5$ of them (3.47\%) underwent a repair by either retrorectus ( 2 patients) or component separation techniques ( 3 patients). There were no complications related to the previous mesh insertion. Even in 2 cases, the prophylactic mesh was not even discerned during surgical repair. The other 8 patients remain asymptomatic and do not want further surgery. No chronic seromas or foreign body reaction have been observed. None of the patients have developed chronic pain.

In the comparative analysis, obesity was a risk factor for IH ( $p=0.05)$ (Figure 6$)$. The incidence of IH was also statistically different in the operative status: $19.4 \%(7 / 36)$ in emergency surgeries and 5.55\% (6/108) in elective procedures $(p=0.01$; RR 4.99: 1.15-21.5). Survival plot after logrank test affirmed these differences $(p=0.031)$ in operative status but not in obesity (BMI $\geq$ 30) $(p=0.081)$ (Figure 7). There were no statistical differences regarding any other preoperative, intraoperative or postoperative variables (Table 3).

\section{DISCUSSION}

To date the most widely accepted, albeit still controversial, surgical techniques to reduce the incidence of $\mathrm{IH}$ are the use of small bites technique to close the linea alba $[19,20]$ and mesh augmentation procedure [2]. In the latest European Hernia Society (EHS) guidelines both methods were given only a weak recommendation [12]. Three recent systematic reviews have shown that mesh prophylaxis reduces the risk of developing an $\mathrm{IH}$ by $85 \%[9,21,22]$. Subgroup 
analyses confirmed this benefit whether the mesh was placed in an onlay, retrorectus, or preperitoneal location. However, there is no data available on more than 2 years follow-up.

This study shows that the use of PMP in ML, in elective and emergency surgery, to prevent incisional hernias is effective and safe in the long term. At mean follow up of 5 years the incisional hernia rate was $5 \%$ for elective and $16 \%$ for emergency surgery, with an overall incidence of $9 \%$.

Which mesh type to use and the best anatomical layer to place the mesh within the abdominal wall has not been determined. We chose a very large-pore mesh because these low-density polypropylene meshes tolerated contaminated fields in our previous experimental model of contamination [23], and was satisfactorily applied in our randomized controlled trial (RCT), that included patients in emergency situation and contaminated settings [14].

Polypropylene meshes have been commonly used to prevent $\mathrm{IH}$ following abdominal aortic aneurysm surgery, obesity surgery or colorectal surgery [24-27]. A few studies have been published on the use of biological, composite and biosynthetic meshes to prevent IH but currently there is little data to support their use [28-30]. The use of other synthetic meshes made from either polyester or polyvinylidene fluoride has not been described.

In the published studies, there is no difference in surgical sites occurrences with regard to which layer of the abdominal wall the mesh is placed, with the exception of seroma development. This is observed more frequently in patients that have an onlay compared with sublay mesh reinforcement [3]. We present $13 \%$ cases of seromas, which are consistent with other reported results with rates of up to $18 \%$ [31]. There were no reported clinically relevant outcomes in relation to the seromas in our series. Our preference is to use a subcutaneous drain to reduce the incidence of seroma formation but we accept there is no clear evidence to support its use [32]. One study has shown that fibrin sealant may reduce seroma formation following onlay mesh reinforcement [33], but this was not confirmed in the PRIMA trial [3]. 
The PRIMA study, a multicentre, double-blind, randomised controlled trial, showed there was no difference in IH rate whether mesh was placed in the onlay or sublay position [3]. We recommend the onlay placement of mesh as it is less technically complex and requires less operative time than the sublay or preperitoneal approach. Sublay mesh placement in the retrorectus plane (the Rives-Stoppa technique) can pose a significant technical challenge and add operative time that may lead to poor uptake among surgeons in the prophylactic setting [9]. Prophylactic onlay mesh placement offers a relatively easy, generalizable technique for all patients undergoing midline laparotomy, for surgeons of all specialties, including vascular surgery, urology and gynaecology [3].

Currently we use long-term resorbable sutures to fix the mesh, but accept other techniques for mesh fixation, such as the use of glues or staples, may have a role, particularly in reducing operative time. However, we do not believe their use would obtain better results in terms of morbidity or IH formation.

There are concerns about the long term problems associated with mesh implantation [34]. In our series, there were only two cases of chronic infection requiring mesh removal. Although using general anesthesia, the surgical removal of the mesh was simple and did not produce IH. This seems a reasonable price to pay for overall IH rates under $10 \%$. Interestingly, the use of PMP did not cause any problems in patients requiring surgery at a later date for other causes. The surgical repair of IH that developed despite PMP was not modified by the presence of the onlay mesh, that was not even noticed in two cases.

One other study has reported the use of PMP in emergency surgery to prevent IH [7]. The authors retrospectively assessed 51 emergency operations with 1-year follow-up. They reported a $6 \% \mathrm{IH}$ rate. Although our IH rate after emergency surgery was $19 \%$, we believe this may, at least in part, be due to our longer follow up. On log rank testing we have shown that emergency surgery is a risk factor for the development of $\mathrm{IH}: 5$ times higher. 
Additionally patients with BMI equal to or higher than $27 \mathrm{~kg} / \mathrm{m}^{2}$ have a more than $30 \%$ chance of developing IH after ML [35]. We have also observed that a higher level of BMI is a risk factor for the development of $\mathrm{IH}$, even when a prophylactic mesh is used. Although we accept this was not confirmed on log-rank testing.

Nonetheless, our study has some important limitations. Due to our inclusion criteria we assessed a heterogeneous population in terms of baseline characteristics and operations performed. The indication to perform PMP was not standardized but based on the judgement of the surgeon responsible for the patient, but our data may be considered a truly representative every-day scenario. Another interesting point would have been to also consider the incidence of parastomal hernias even in case of temporary ones. We believe that future studies must take this into account. Assessment of IH by CT was only made in oncological patients or in case of clinical doubts in non-oncological. If all patients had undergone a CT scan, a slightly higher number of IH might have potentially been identified. Although, we did not include evaluation of QoL in our study, we have not found that abdominal wall pain was an impairment symptom in our patients. We have not considered the cost-effectiveness in our study, which would be an interesting point, since a recent cost-utility analysis revealed that PMP is less costly and overall more effective than primary suture closure [36].

\section{CONCLUSIONS}

The published data to date has shown that PMP at the time of closing midline laparotomies is safe and effective at reducing the rate of incisional hernias up to 2 years follow-up. This study shows that its use, in elective and emergency surgery, is safe and effective in the long term as well. 


\section{DECLARATION OF CONFLICT OF INTEREST}

CS declares no conflict of interest.

DM declares no conflict of interest.

EJ declares no conflict of interest.

PL declares no conflict of interest.

AR declares no conflict of interest.

LAB declares conflict of interest not directly related to the submitted work (GORE).

JLM declares conflict of interest not directly related to the submitted work (GORE).

EG declares no conflict of interest.

CJ declares no conflict of interest.

MAGU declares conflict of interest not directly related to the submitted work (COVIDIEN, B BRAUN, GORE and MEDTRONIC).

\section{Author's contribution to the Work:}

MAGU, LAB and JLM conceived of the presented idea. MAGU, CS and DM designed the study. CS, EJ, CJ and PL collected data from the registry and AR verified the analytical methods. MAGU and EG encouraged CS, DM and EJ to investigate and supervised the findings of this work. All authors discussed the results and contributed to the final manuscript. 


\section{REFERENCES}

1. Veljkovic R, Protic M, Gluhovic A, Potic Z, Milosevic Z, Stojadinovic A (2010) Prospective clinical trial of factors predicting the early development of incisional hernia after midline laparotomy. J Am Coll Surg 210(2):210-9.

2. Rhemtulla IA, Messa CA, Enriquez FA, Hope WW, Fischer JP (2018) Role of prophylactic mesh placement for laparotomy and stoma creation. Surg Clin North Am 98(3):471-481.

3. Jairam AP, Timmermans L, Eker HH, Pierik REGJM, van Klaveren D, Steyerberg EW, et al (2017) Prevention of incisional hernia with prophylactic onlay and sublay mesh reinforcement versus primary suture only in midline laparotomies (PRIMA): 2-year follow-up of a multicentre, double-blind, randomised controlled trial. Lancet 390(10094):567-76.

4. Muysoms FE, Detry O, Vierendeels T, Huyghe M, Miserez M, Ruppert M, et al (2016) Prevention of Incisional Hernias by Prophylactic Mesh-augmented Reinforcement of Midline Laparotomies for Abdominal Aortic Aneurysm Treatment: A Randomized Controlled Trial. Ann Surg 263(4):638-45.

5. Alnassar S, Bawahab M, Abdoh A, Guzman R, Al Tuwaijiri T, Louridas G (2012) Incisional hernia postrepair of abdominal aortic occlusive and aneurysmal disease: five-year incidence. Vascular 20(5):273-7.

6. Mingoli A, Puggioni A, Sgarzini G, Luciani G, Corzani F, Ciccarone F, et al (1999) Incidence of incisional hernia following emergency abdominal surgery. Ital J Gastroenterol Hepatol 31(6):449-53. 
7. Argudo N, Pereira JA, Sancho JJ, Membrilla E, Pons MJ, Grande L (2014) Prophylactic synthetic mesh can be safely used to close emergency laparotomies, even in peritonitis. Surgery 156(5):1238-44.

8. Burger JWA, Luijendijk RW, Hop WCJ, Halm JA, Verdaasdonk EGG, Jeekel J (2004) Longterm follow-up of a randomized controlled trial of suture versus mesh repair of incisional hernia. Ann Surg 240(4):578-83-5.

9. Borab ZM, Shakir S, Lanni MA, Tecce MG, MacDonald J, Hope WW, et al (2017) Does prophylactic mesh placement in elective, midline laparotomy reduce the incidence of incisional hernia? A systematic review and meta-analysis. Surgery 161(4):1149-63.

10. Gillion J-F, Sanders D, Miserez M, Muysoms F (2016) The economic burden of incisional ventral hernia repair: a multicentric cost analysis. Hernia 20(6):819-30.

11. Itatsu K, Yokoyama Y, Sugawara G, Kubota H, Tojima Y, Kurumiya Y, et al (2014) Incidence of and risk factors for incisional hernia after abdominal surgery. Br J Surg 101(11):143947.

12. Muysoms FE, Antoniou SA, Bury K, Campanelli G, Conze J, Cuccurullo D, et al (2015) European Hernia Society guidelines on the closure of abdominal wall incisions. Hernia 19(1):1-24.

13. Timmermans L, de Goede B, Eker HH, van Kempen BJH, Jeekel J, Lange JF (2013) Metaanalysis of primary mesh augmentation as prophylactic measure to prevent incisional hernia. Dig Surg 30(4-6):401-9.

14. García-Ureña MÁ, López-Monclús J, Hernando LAB, Montes DM, Valle de Lersundi AR, Pavón CC, et al (2015) Randomized controlled trial of the use of a large-pore polypropylene mesh to prevent incisional hernia in colorectal surgery. Ann Surg 261(5):876-81. 
15. STROBE Statement: https://www.strobe-statement.org/index.php?id=strobe-home. Accesed 30 Oct 2017.

16. Berríos-Torres SI, Umscheid CA, Bratzler DW, Leas B, Stone EC, Kelz RR, et al (2017) Centers for Disease Control and Prevention Guideline for the prevention of surgical site infection, 2017. JAMA Surg 152(8):784.

17. Treede R-D, Rief W, Barke A, Aziz Q, Bennett MI, Benoliel R, et al (2015) A classification of chronic pain for ICD-11. Pain 156(6):1.

18. Muysoms FE, Miserez M, Berrevoet F, Campanelli G, Champault GG, Chelala E, et al (2009) Classification of primary and incisional abdominal wall hernias. Hernia 13(4):40714.

19. Millbourn D, Cengiz Y, Israelsson LA (2009) Effect of Stitch Length on Wound Complications After Closure of Midline Incisions. Arch Surg 144(11):1056.

20. Deerenberg EB, Harlaar JJ, Steyerberg EW, Lont HE, van Doorn HC, Heisterkamp J, et al (2015) Small bites versus large bites for closure of abdominal midline incisions (STITCH): a double-blind, multicentre, randomised controlled trial. Lancet 386(10000):1254-60.

21. Wang X-C, Zhang D, Yang Z-X, Gan J-X, Yin L-N (2017) Mesh reinforcement for the prevention of incisional hernia formation: a systematic review and meta-analysis of randomized controlled trials. J Surg Res 209:17-29.

22. Payne R, Aldwinckle J, Ward S (2017) Meta-analysis of randomised trials comparing the use of prophylactic mesh to standard midline closure in the reduction of incisional herniae. Hernia 21(6):843-53.

23. Díaz-Godoy A, García-Ureña MA, López-Monclús J, Vega V, Melero D, Erquinigo N (2011) Searching for the best polypropylene mesh to be used in bowel contamination. Hernia 15(2):173-9. 
24. Bevis PM, Windhaber RAJ, Lear PA, Poskitt KR, Earnshaw JJ, Mitchell DC (2010) Randomized clinical trial of mesh versus sutured wound closure after open abdominal aortic aneurysm surgery. Br J Surg 97(10):1497-502.

25. Strzelczyk JM, Szymański D, Nowicki ME, Wilczyński W, Gaszynski T, Czupryniak L (2006) Randomized clinical trial of postoperative hernia prophylaxis in open bariatric surgery. $\mathrm{Br}$ J Surg 93(11):1347-50.

26. Abo-Ryia MH, El-Khadrawy OH, Abd-Allah HS (2013) Prophylactic Preperitoneal Mesh Placement in Open Bariatric Surgery: a Guard Against Incisional Hernia Development. Obes Surg 23(10):1571-4.

27. Hidalgo MP, Ferrero EH, Ortiz MA, Castillo JMF, Hidalgo AG (2011) Incisional hernia in patients at risk: can it be prevented? Hernia 15(4):371-5.

28. Bali C, Papakostas J, Georgiou G, Kouvelos G, Avgos S, Arnaoutoglou E, et al (2015) A comparative study of sutured versus bovine pericardium mesh abdominal closure after open abdominal aortic aneurysm repair. Hernia 19(2):267-71.

29. Llaguna $\mathrm{OH}$, Avgerinos D V, Nagda P, Elfant D, Leitman IM, Goodman E (2011) Does prophylactic biologic mesh placement protect against the development of incisional hernia in high-risk patients? World J Surg 35(7):1651-5.

30. Söderbäck H, Mahteme H, Hellman P, Sandblom G (2016) Prophylactic resorbable synthetic mesh to prevent wound dehiscence and incisional hernia in high-risk laparotomy: A pilot study of Using TIGR Matrix Mesh. Front Surg 3:28.

31. Timmermans L, Eker HH, Steyerberg EW, Jairam A, de Jong D, Pierik EGJM, et al (2015) Short-term results of a randomized controlled trial comparing primary suture with primary glued mesh augmentation to prevent incisional hernia. Ann Surg 261(2):276-81.

32. Gurusamy KS, Allen VB (2013) Wound drains after incisional hernia repair. Cochrane 
Database Syst Rev 17 (12):CD005570.

33. Fortelny RH, Petter-Puchner AH, Glaser KS, Redl H (2012) Use of fibrin sealant (Tisseel/Tissucol) in hernia repair: a systematic review. Surg Endosc 26 (7):1803-12.

34. Plencner M, East B, Tonar Z, Otáhal M, Prosecká E, Rampichová M, et al (2014) Abdominal

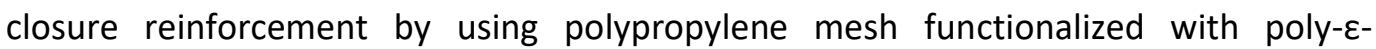
caprolactone nanofibers and growth factors for prevention of incisional hernia formation. Int J Nanomedicine 9:3263-77.

35. Seiler CM, Bruckner T, Diener MK, Papyan A, Golcher H, Seidlmayer C, et al (2009) Interrupted or continuous slowly absorbable sutures for closure of primary elective midline abdominal incisions. Ann Surg 249(4):576-82.

36. Fischer JP, Basta MN, Wink JD, Krishnan NM, Kovach SJ (2015) Cost-utility analysis of the use of prophylactic mesh augmentation compared with primary fascial suture repair in patients at high risk for incisional hernia. Surgery 158(3):700-11. 


\section{TABLE LEGENDS}

Table 1. Descriptive characteristics of patients

Table 1 Descriptive characteristics of patients

Demographics

Age (years), median (Q25-Q75)

Sex (M:F)

BMI $\left(\mathrm{kg} / \mathrm{m}^{2}\right)$, median (Q25-Q75)

Comorbidities $(n, \%)$

Smoking

Heart disease

Hypertension

Chronic obstructive pulmonary disease

Diabetes

Obesity

Previous history of cancer

Diagnosis $(n, \%)$

Acute diverticulitis

Colorrectal cancer (complicated)

Colorrectal cancer (no complicated)

Recurrence of colorrectal cancer

Intestinal obstruction

Complications of previous surgeries

Ischemia

Abdominal sepsis

Others
$68(62-77)$

$96: 76$

$28.6(25.2-30.8)$

$71(41.3 \%)$

$53(30.8 \%)$

$94(54.7 \%)$

$24(14 \%)$

$38(22.1 \%)$

$44(25.6 \%)$

$34(19.8 \%)$

$6(3.5 \%)$

$7(4.1 \%)$

$121(70.3 \%)$

$3(1.7 \%)$

$17(9.9 \%)$

$3(1.7 \%)$

$1(0.6 \%)$

$4(2.3 \%)$

$10(5.8 \%)$ 
Treatment $(n, \%)$

Left hemicolectomy/sigmoidectomy

$45(26.2 \%)$

Right hemicolectomy

$39(22.7 \%)$

Anterior resection of rectum

$30(17.4 \%)$

TotaV/subtotal colectomy

$16(9.3 \%)$

Small bowel resection

$9(5.2 \%)$

Abdominoperineal resection

$8(4.7 \%)$

Exploratory laparotomy

$12(7 \%)$

Adhesiolysis

$11(6.4 \%)$

Operative status $(n, \%)$

Elective

$129(75 \%)$

Urgent

$43(25 \%)$

Wound classification $(n, \%)$

Clean

$11(6.4 \%)$

Clean-contaminated

$145(85 \%)$

Contaminated

$2(1.2 \%)$

Dirty

$14(8.1 \%)$

Stomas $(n, \%)$

Colostomies

$29(16.9 \%)$

Ileostomies

$19(11 \%)$

Surgical site occurrences $(n, \%)$

Surgical site infections (superficial)

$17(9.9 \%)$

Surgical site infections (deep)

$2(1.1 \%)$

Seromas

$23(13.4 \%)$

Organ/space infections

$9(5.2 \%)$

Table 2. Incidence of IH in different groups of patients.

Table 2 Incidence of $\mathrm{IH}$ in different groups of patients

\begin{tabular}{lllllll}
\hline Incidence & Total & \multicolumn{2}{l}{ Priority of surgery } & & \multicolumn{2}{l}{ Type of surgery } \\
\cline { 6 - 7 } \cline { 6 - 7 } & & Emergency & Elective & & $\begin{array}{l}\text { Oncologi- } \\
\text { cal }\end{array}$ & $\begin{array}{l}\text { Non- } \\
\text { oncolog- } \\
\text { ical }\end{array}$ \\
\hline Number & $13 / 144$ & $7 / 36$ & $6 / 108$ & $8 / 113$ & $5 / 31$ \\
$\%$ & 9.02 & 19.4 & 5.55 & & 7 & 16.1 \\
\hline
\end{tabular}


Table 3. Statistical analysis of variables in the study.

\begin{tabular}{|c|c|c|c|c|}
\hline \multirow[t]{2}{*}{ Variable } & \multicolumn{2}{|l|}{$\mathrm{IH}$} & \multirow{2}{*}{\multicolumn{2}{|c|}{$\begin{array}{l}p \text { (Mann-Withney } \\
U)\end{array}$}} \\
\hline & Yes & No & & \\
\hline BMI $\left(\mathrm{kg} / \mathrm{m}^{2}\right)$ & $30.7(28.6-32.4)$ & $28(24.9-30.5)$ & 0.050 & \\
\hline Age (years) & $68(60-73)$ & $69(62-77)$ & 0.620 & \\
\hline \multicolumn{3}{|l|}{ Variable } & $p\left(\chi^{2}\right)$ & $p$ (log-rank $)^{*}$ \\
\hline Sex (e. g. men) & $7(8.6 \%)$ & $79(91.4 \%)$ & 1.000 & 0.921 \\
\hline Obesity & $6(15.4 \%)$ & $33(84.6 \%)$ & 0.080 & 0.049 \\
\hline Smoking & $6(9.5 \%)$ & $57(90.5 \%)$ & 0.763 & 0.280 \\
\hline Cardiopathy & $4(10 \%)$ & $36(90 \%)$ & 0.756 & 0.780 \\
\hline Hypertension & $7(9.3 \%)$ & $68(90.7 \%)$ & 1.000 & 0.859 \\
\hline $\mathrm{COPD}^{2}$ & $2(9.5 \%)$ & $19(90.5 \%)$ & 1.000 & 0.661 \\
\hline Diabetes & $1(3.4 \%)$ & $28(96.6 \%)$ & 0.467 & 0.433 \\
\hline \multicolumn{3}{|l|}{ Wound classification } & 0.094 & 0.715 \\
\hline Clean & $2(22.2 \%)$ & $7(77.8 \%)$ & & \\
\hline Clean contaminated & $9(7.4 \%)$ & $113(92.6 \%)$ & & \\
\hline Contaminated & $1(50 \%)$ & $1(50 \%)$ & & \\
\hline Dirty & $1(9.1 \%)$ & $10(90.9 \%)$ & & \\
\hline \multicolumn{3}{|l|}{ Type of surgery } & 0.626 & 0.278 \\
\hline Left hemicolectorny/sigmoidectomy & $4(9.3 \%)$ & $30(90.7 \%)$ & & \\
\hline Right hemicolectomy & $3(9.1 \%)$ & $30(90.9 \%)$ & & \\
\hline Anterior resection of rectum & $1(4.2 \%)$ & $23(95.8 \%)$ & & \\
\hline TotaV subtotal colectomy & $0(0 \%)$ & $13(100 \%)$ & & \\
\hline Small bowel resection & $1(16.7 \%)$ & $5(83.3 \%)$ & & \\
\hline Abdominoperineal resection & $0(0 \%)$ & $6(100 \%)$ & & \\
\hline Exploratory laparotomy & $1(12.5 \%)$ & $7(87.5 \%)$ & & \\
\hline Adhesiolysis & $2(22.2 \%)$ & $7(77.8 \%)$ & & \\
\hline Urgent surgery & $7(19.4 \%) 7(19.4 \%)$ & $29(80.6 \%) 29(80.6 \%)$ & 0.010 & 0.031 \\
\hline \multicolumn{3}{|l|}{ Stoma formation } & 0.491 & 0.249 \\
\hline Ileostomy & $0(0 \%)$ & $18(100 \%)$ & & \\
\hline Colostomy & $3(13 \%)$ & $20(87 \%)$ & & \\
\hline
\end{tabular}

${ }^{*}$ Applicable in cathegorical variables ${ }^{2}$ Chronic obstructive pulmonary desease 


\section{FIGURE LEGENDS}

Figure 1. Flow diagram of patients.

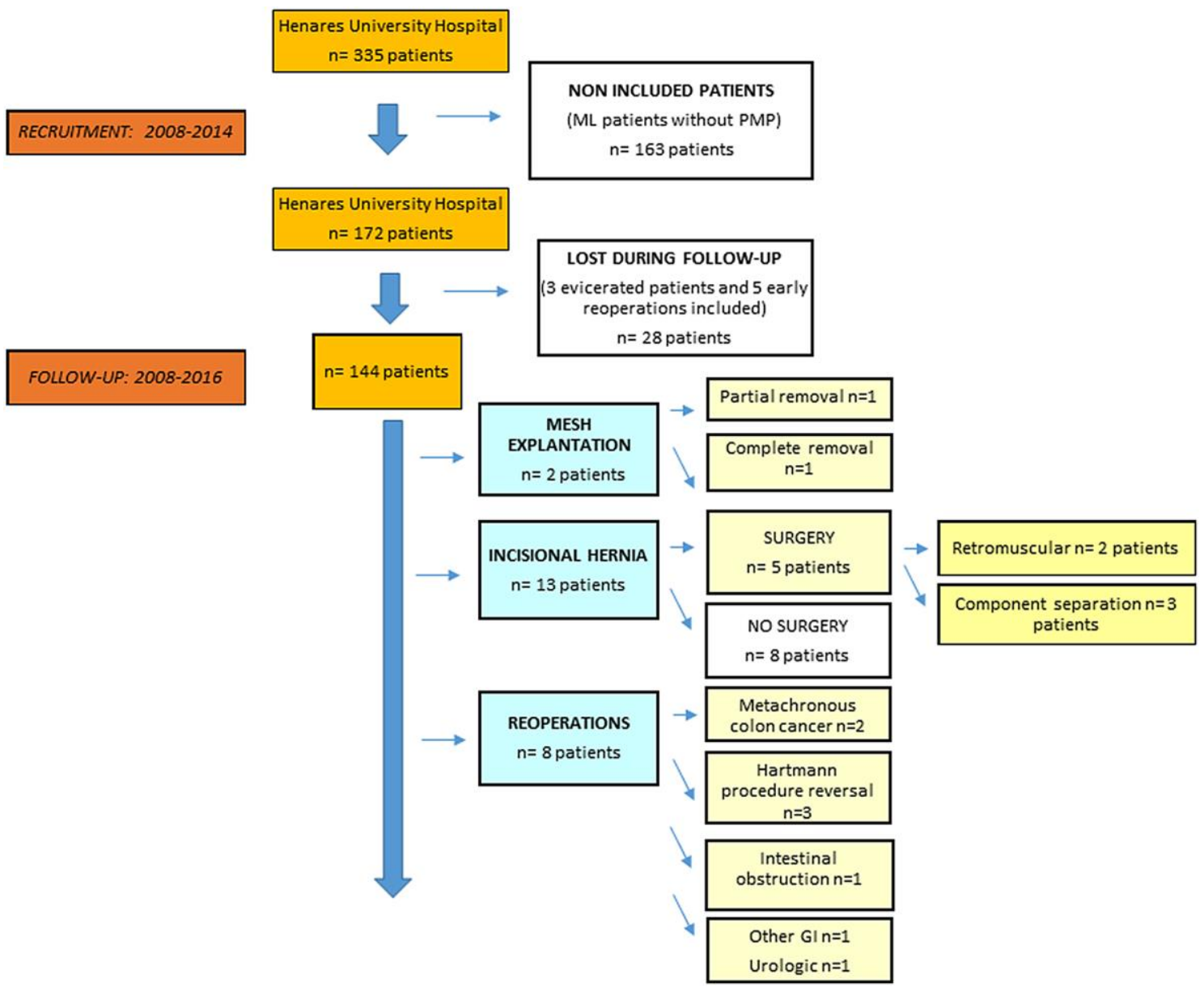

Figure 2. Case of mesh explantation: A) Chronic infection; B) Mesh exposure; C) Mesh removal; D) Mesh explanted.

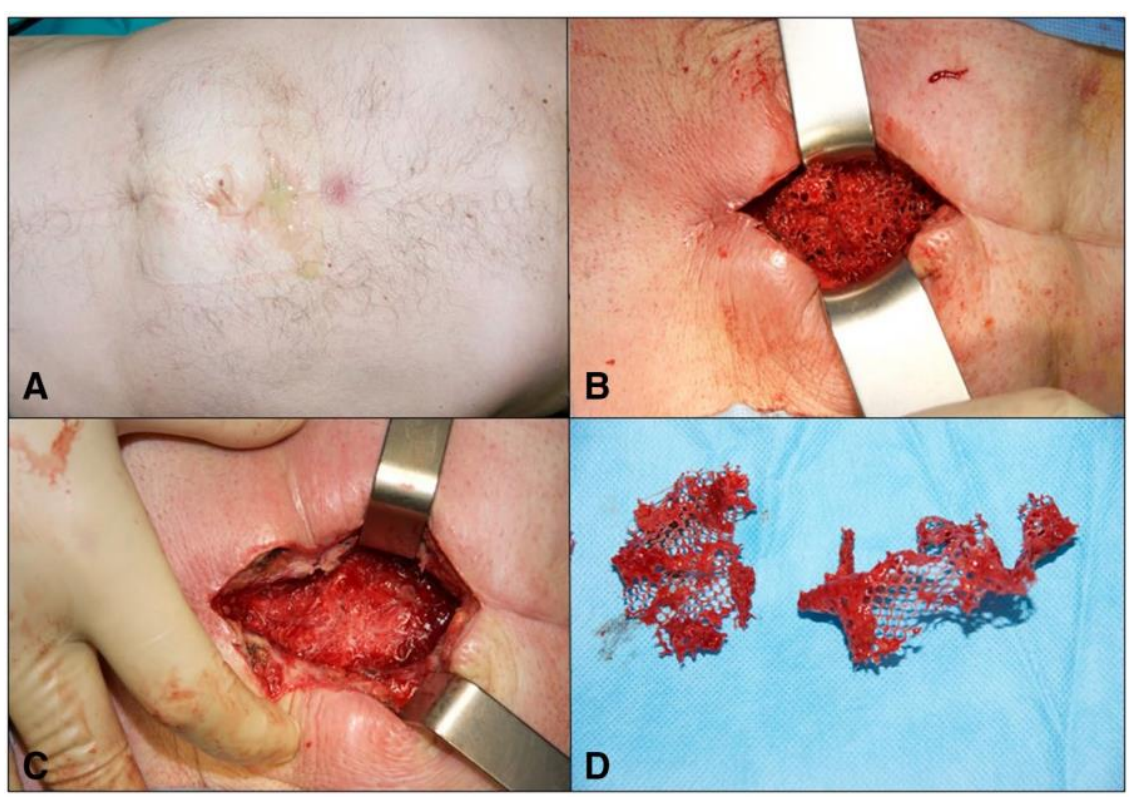


Figure 3. Follow-up of patients. Box-plot.

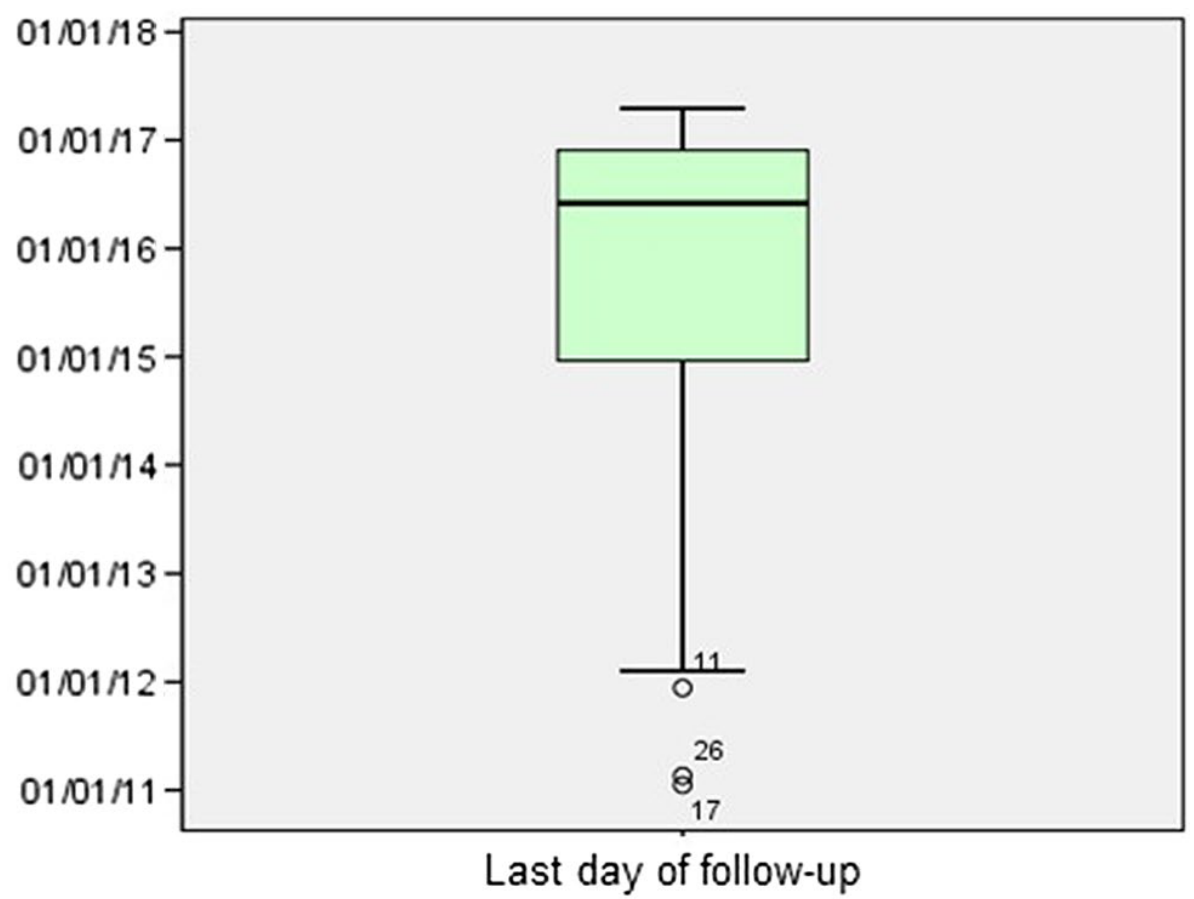

Figure 4. Estimated freedom of incisional hernia curves.

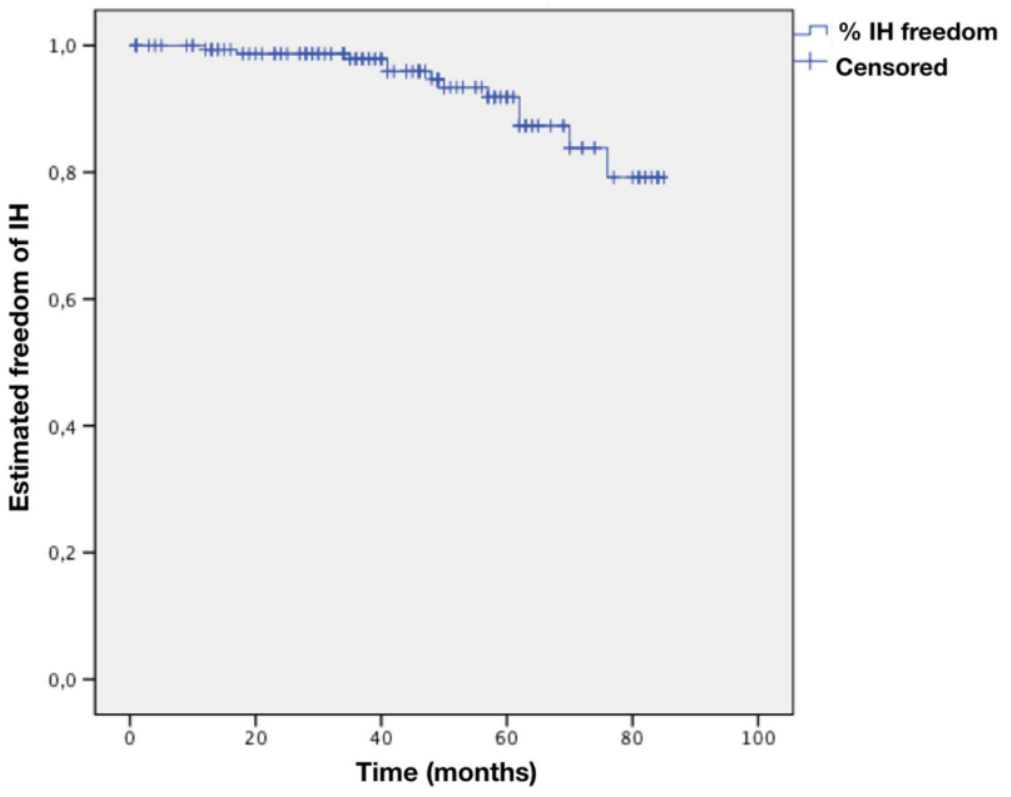


Figure 5. Intraoperative findings during reoperation of a PMP patient.

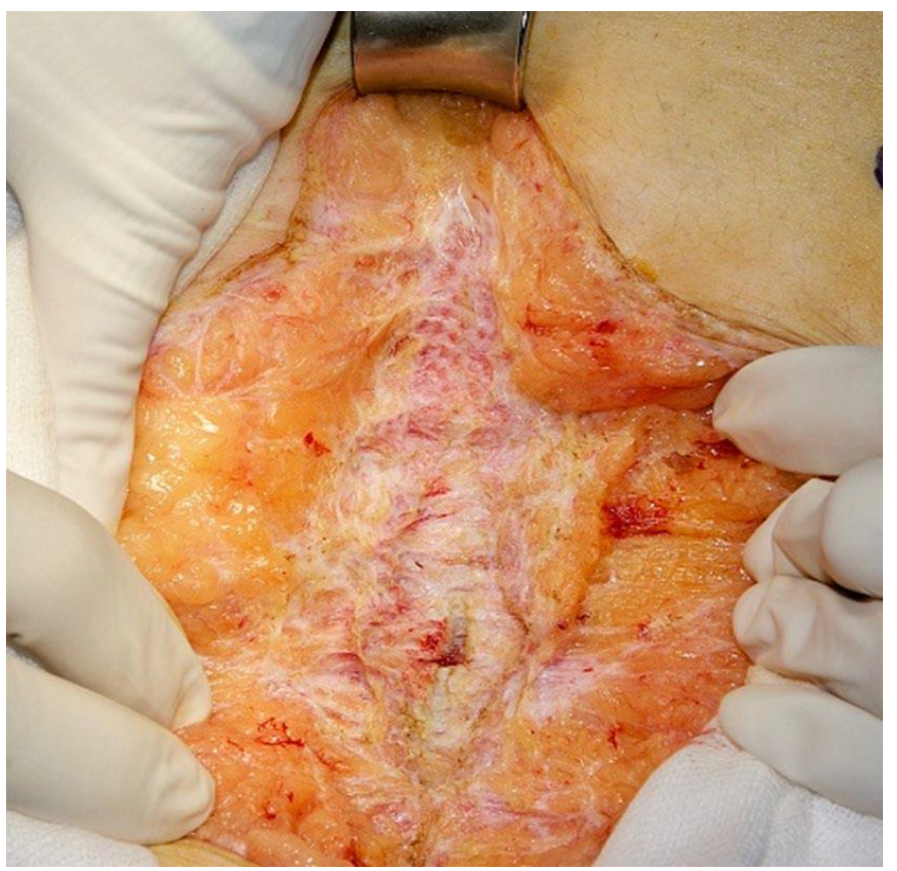

Figure 6. Development of incisional hernia by body mass index.

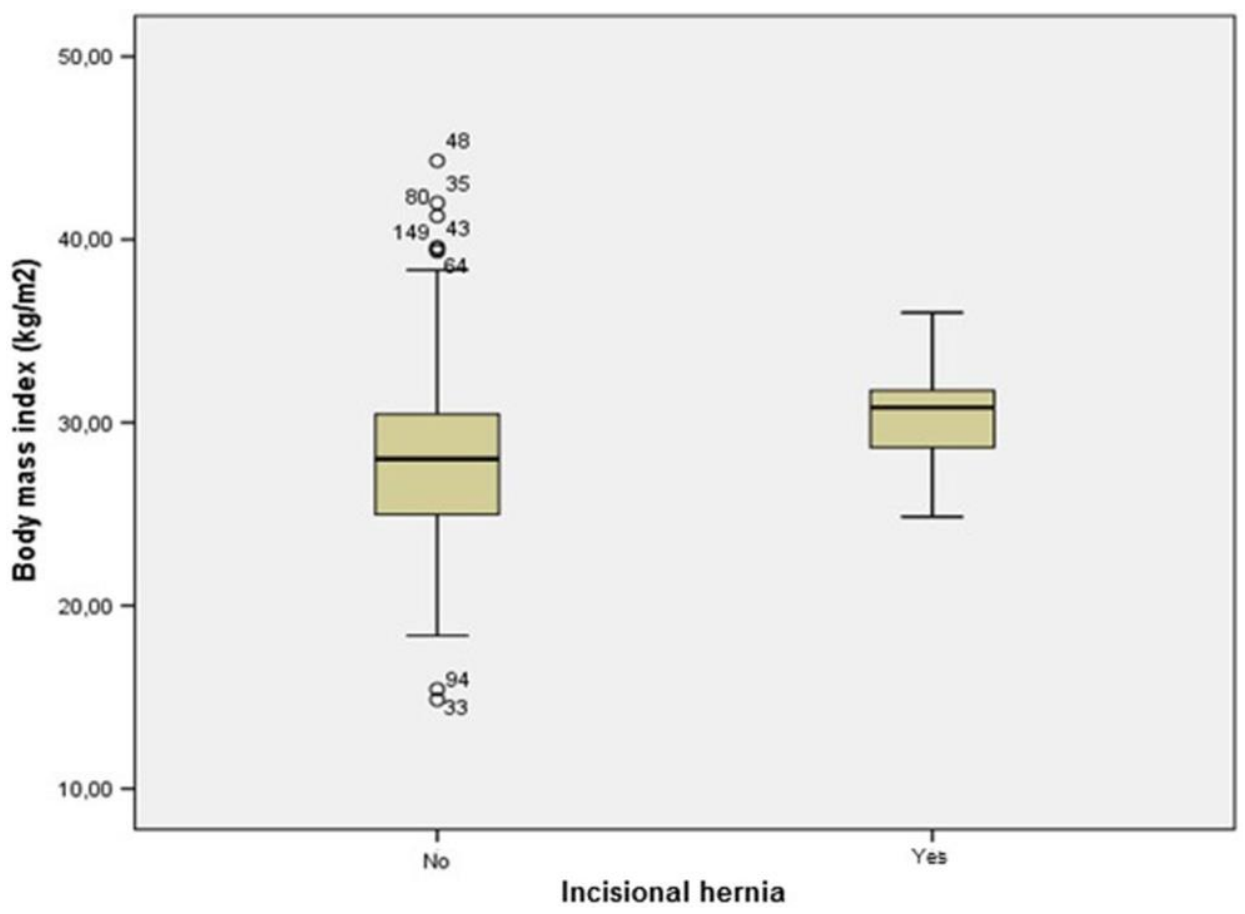


Figure 7. Estimated freedom of incisional hernia curves by operative status.

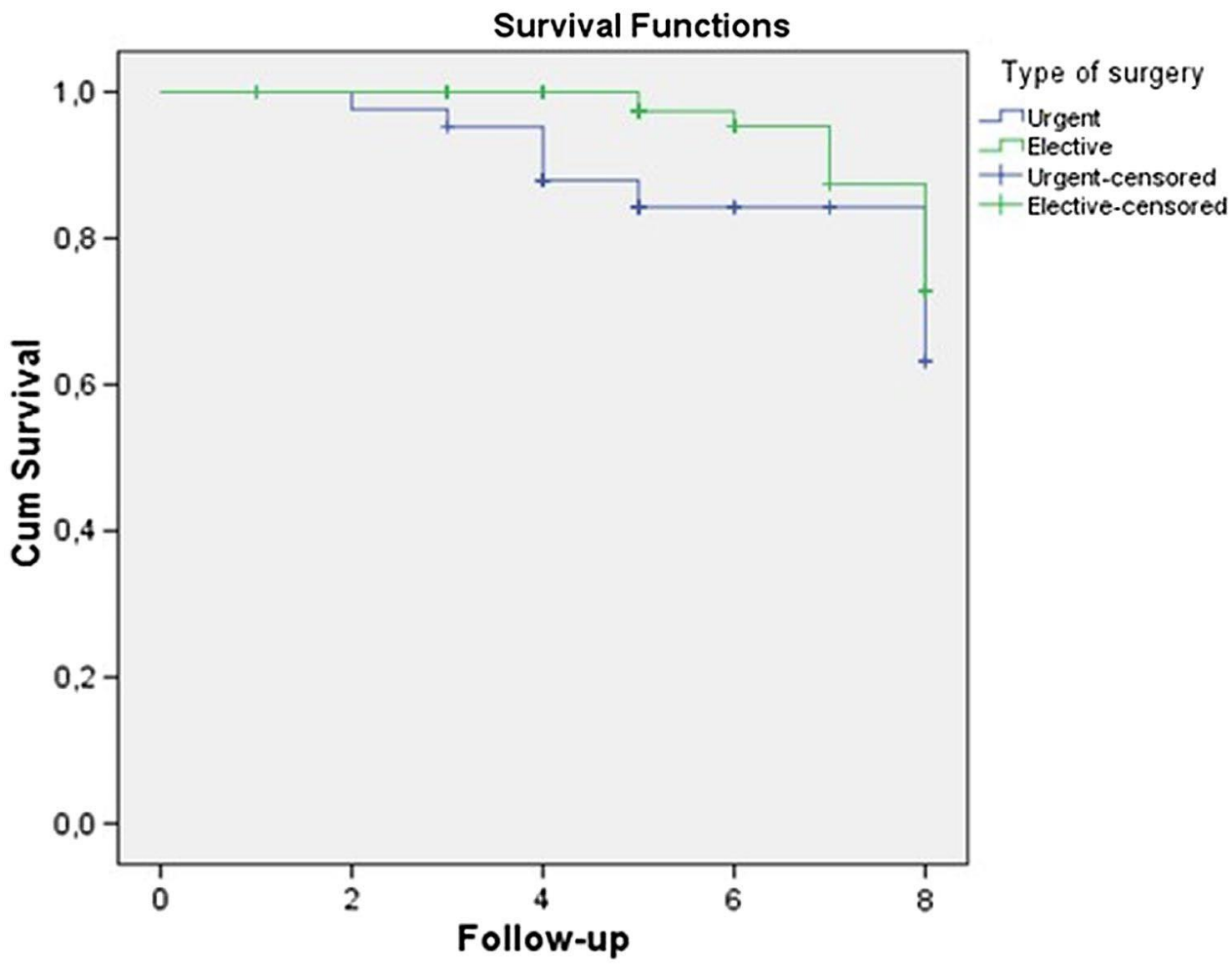




\section{ABBREVIATIONS}

BMI: Body mass index

COPD: Chronic obstructive pulmonary disease

CT: computed tomographic scan

IH: Incisional hernia

ML: Midline laparotomy

PMP: Prophylactic mesh placement

QoL: Quality of life

RCT: Randomized controlled trial

RR: Risk ratio

SPSS: Statistical Package for the Social Sciences

SSI: Surgical site infection 